\title{
Un deuxième ligand pour le récepteur du ciliary neurotrophic factor (CNTF) facteur de survie des motoneurones
}

$\mathbf{L}$ e ciliary neurotrophic factor (CNTF) est une cytokine qui permet la différenciation et la survie de différents types cellulaires du système nerveux central et du système nerveux périphérique $(\mathrm{m} / \mathrm{s}$ 1996, n², p. 246). D'abord identifié chez le poulet comme un facteur de survie pour les neurones parasympathiques des ganglions ciliaires, le CNTF a ensuite été caractérisé chez les mammifères (Tableau I) [1, 2]. In vitro, on mentionnera en particulier son action sur la survie des neurones dopaminergiques de la substance noire, ainsi que sur les motoneurones. Le CNTF agit aussi sur la différenciation des neurones vers un phénotype cholinergique. In vivo, il protège les motoneurones de la dégénérescence consécutive à une axotomie [3], et retarde le développement de déficits moteurs chez différentes souches de souris présentant des déficiences neuromusculaires congénitales $[4,5]$. Son action ne se limite pas aux tissus nerveux, et le CNTF est aussi un facteur trophique pour les muscles squelettiques, notamment lors de dénervations ou au cours du vieillissement [6, 7]. Ces observations ont suscité de nombreux espoirs quant à son utilisation comme agent thérapeutique dans les maladies neurodégénératives.

L'identification d'une seconde cytokine présentant des activités voisines de celles décrites pour le CNTF n'est pas une totale surprise, et plusieurs éléments le laissaient pressentir : la protéine CNTF est dépourvue de séquence de sécrétion, et les cellules gliales, qui le synthétisent ne l'excréteraient que lors d'épisodes trauma- tiques ou d'états de souffrance des tissus nerveux. Par ailleurs, 2,3\% de la population japonaise présente une mutation homozygote qui inactive le gène du CNTF, mais n'entraîne aucune pathologie neurologique, y compris chez le sujet âgé [8]. De même, l'inactivation du gène $d u$ CNTF chez la souris n'induit pas de défaut majeur de la motricité $(\mathrm{m} / \mathrm{s}$ 1996, $n^{\circ} 2, p .945$ ), contrairement à celle de la sous-unité réceptrice (CNTFR) permettant la fixation membranaire du CNTF, qui, elle, est

\begin{tabular}{|ll|}
\hline \multicolumn{2}{|c|}{ Tableau I } \\
ACTIVITÉS BIOLOGIQUES \\
DU CNTF
\end{tabular}

associée à un phénotype très sévère avec mort périnatale des animaux [9]. Les souriceaux ne peuvent alors se nourrir et présentent des pertes conséquentes au niveau des populations de neurones moteurs. Cet important contraste entre le phénotype silencieux des souris $\mathrm{CNTF}^{-/}$et la gravité de celui des souris $C N T F R^{-1-}$ suggère l'existence d'un deuxième ligand du récepteur du CNTF, présent notamment lors de la vie embryonnaire, et jouant un rôle prépondérant dans le développement de l'axe neuromusculaire.

Pour définir la nature de ce deuxième ligand, il est important de rappeler que le CNTF appartient à la famille des cytokines neuropoïétiques dites «de l'interleukine-6 " (IL-6) [10]. Cette famille de cytokines comprend également le leukemia inhibitory factor (LIF), l'interleukine-11 (IL-11), l'oncostatine M (OSM), la cardiotrophine-1 (CT-1) et la cardiotrophin-like cytokine (CLC), plus récemment décrite [11]. Cette dernière est également connue sous le nom de novel neurotrophin-1 et B-cell stimulating factor-3 [12].

D'un point de vue structural, les cytokines de la famille de l'IL-6/CNTF sont constituées de 4 hélices $\alpha$ disposées de façon antiparallèle deux à deux et reliées entre elles par trois boucles. Elles agissent sur leurs cibles par l'intermédiaire de récepteurs membranaires multimériques [13]. La sous-unité réceptrice de fixation du CNTF sur la membrane, le CNTFR, présente des homologies de structure avec le récepteur de l'interleukine-6 (figure 1). On notera en particulier la présence de deux ponts cys- 


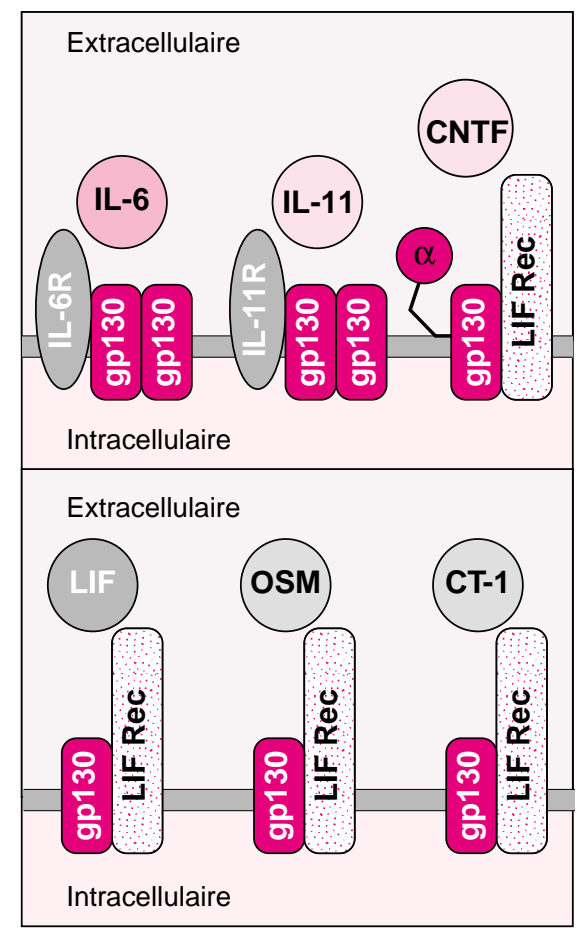

Figure 1. Représentation schématique des récepteurs multimériques pour les cytokines de la famille de I'IL-6. La sous-unité réceptrice gp 130 va former un homodimère (IL-6, IL-11) ou un hétérodimère en s'association avec le récepteur pour le LIF (CNTF, LIF, OSM, CT-1). Pour I'IL-6, I'IL-11 et le CNTF l'association à la membrane impliquera également des chaînes de fixations spécifiques, ou chaînes " $\alpha$ ".

téines dont les positions sont conservées, et la présence d'un petit segment d'acides aminés retrouvé dans la quasi-totalité des récepteurs de cytokines, le motif "Trp-Ser-X-TrpSer». Le CNTFR, de poids moléculaire $72 \mathrm{kDa}$, est un récepteur principalement extra-membranaire qui est inséré dans la membrane cellulaire par l'intermédiaire d'un groupement glycosylphosphatidylinositol [14]. Il est présent essentiellement sur les neurones et les cellules des muscles squelettiques. Les études de la transduction du signal induit par la fixation membranaire du CNTF à son récepteur montrent que les différentes cytokines de la famille de l'IL-6 utilisent les mêmes sous-unités récep- taille, comme le récepteur de signalisation gp130. Ainsi, le récepteur fonctionnel pour le CNTF comprend, outre le CNTFR, la gp 130, commune à l'ensemble des cytokines de la famille, et le récepteur pour le LIF (LIFR) [15] (figure 1). L'association gp130/LIFR est également un héterorécepteur fonctionnel pour des cytokines voisines, comme le LIF, l'oncostatine $\mathrm{M}$ et la cardiotrophine-1 [13]. L'expression membranaire du CNTFR confère au récepteur tripartite une sélectivité et une spécificité de liaison pour le CNTF. La fixation du CNTF sur son récepteur fonctionnel va engendrer des modifications structurales des chaînes réceptrices avec notamment un contact et une dimérisation des chaînes transmembranaires gp130 et LIFR qui vont conduire à un recrutement et à une activation des kinases sous-membranaires associées de la famille "JAK" [16] $\left(\mathrm{m} / \mathrm{s}\right.$ 1998, $n^{\circ} 10$, p. 1129). Les kinases de la famille JAK vont alors phosphoryler différents résidus tyrosine situés sur les portions intracellulaires de gp130 et de LIFR. Une fois phosphorylés, ces résidus serviront de sites d'ancrage pour amorcer les cascades de transduction du signal et amener l'information vers le noyau. On mentionnera la voie dite des protéines «STAT», en particulier STAT3, ainsi que les voies des MAP kinases et de la PI3 kinase [17].

La caractérisation d'un deuxième ligand pour le récepteur du CNTF fait suite à l'identification d'un récepteur soluble de cytokines, le CLF (pour cytokine like-factor-1) présentant des traits structuraux proches de la sous-unité p40 de l'IL-12 [18]. L'IL12 est une cytokine immunomodulatrice formée par l'association de 2 sous-unités : une partie cytokinique, p35, liée de façon covalente à un élément de type récepteur soluble de cytokine, p40 [19]. La similitude structurale entre p40 et CLF nous laissait penser que CLF entrait dans la formation d'une nouvelle cytokine héterodimérique suivant le modèle de l'IL-12. En 1999, deux études ont identifié une nouvelle molécule appartenant à la famille de l'IL-6 et appelée cardiotrophin-like cytokine ou CLC $[11,12]$. Nous avons alors montré que lorsqu'elle est exprimée dans des cellules de mammifères, la cytokine CLC reste dans le compartiment intracellulaire et ce, malgré la présence d'un peptide signal dans sa séquence. Cette situation est analogue à celle décrite pour la sousunité p35 de l'IL-12. En revanche, la co-expression de CLC avec CLF conduit à la formation d'un hétérodimère non-covalent qui est sécrété à l'extérieur de la cellule (figure 2) [20]. Nous avons ensuite démontré que l'héterodimère CLC/CLF se fixe sur le CNTFR. Dans des expériences de compétition utilisant CLC/CLF et CNTF, les deux ligands sont capables de déplacer mutuellement leur fixation sur le récepteur tripartite du CNTF. Par ailleurs, la modélisation en trois dimensions de la partie cytokinique, CLC, et sa comparaison avec le CNTF soulignent la conservation du squelette architectural des deux cytokines (figure 3). On observe notamment la présence sur CLC d'un «site I» et d'un «site III» qui définissent respectivement les zones de contact avec les sous-unités CNTFR et LIFR (13). La zone d'interaction avec gp130 ou « site II » est, comme pour les cytokines voisines, définie par des points de contact multiples dispersés sur une plus large portion de la molécule. Il est important de souligner l'utilisation par CLC de 2 récepteurs de cytokines de type chaînes $\alpha$ (CLF, CNTFR). Cette situation est tout-à-fait nouvelle dans le domaine des cytokines. L'une d'elle, CLF, va régler la sécrétion de la partie cytokinique ; l'autre, CNTFR, va conditionner la réponse biologique à la cytokine. Il est probable que ce mode de régulation de la fonction biologique soit aussi utilisé par d'autres cytokines.

L'ancrage glycolipidique du CNTFR dans la membrane peut-être clivé par une phospholipase pour donner une forme soluble de CNTFR. Celui-ci reste capable de lier le ligand, et l'association CNTF/CNTFR soluble va pouvoir ensuite activer les sousunités réceptrices gp130 et LIFR [21]. D'une façon similaire, nous avons analysé l'aptitude de CLC/CLF à se fixer à la forme soluble de CNTFR. L'interaction entre les trois éléments du complexe est facilement détectable par des expériences de coimmunoprécipitation, mais curieuse- 


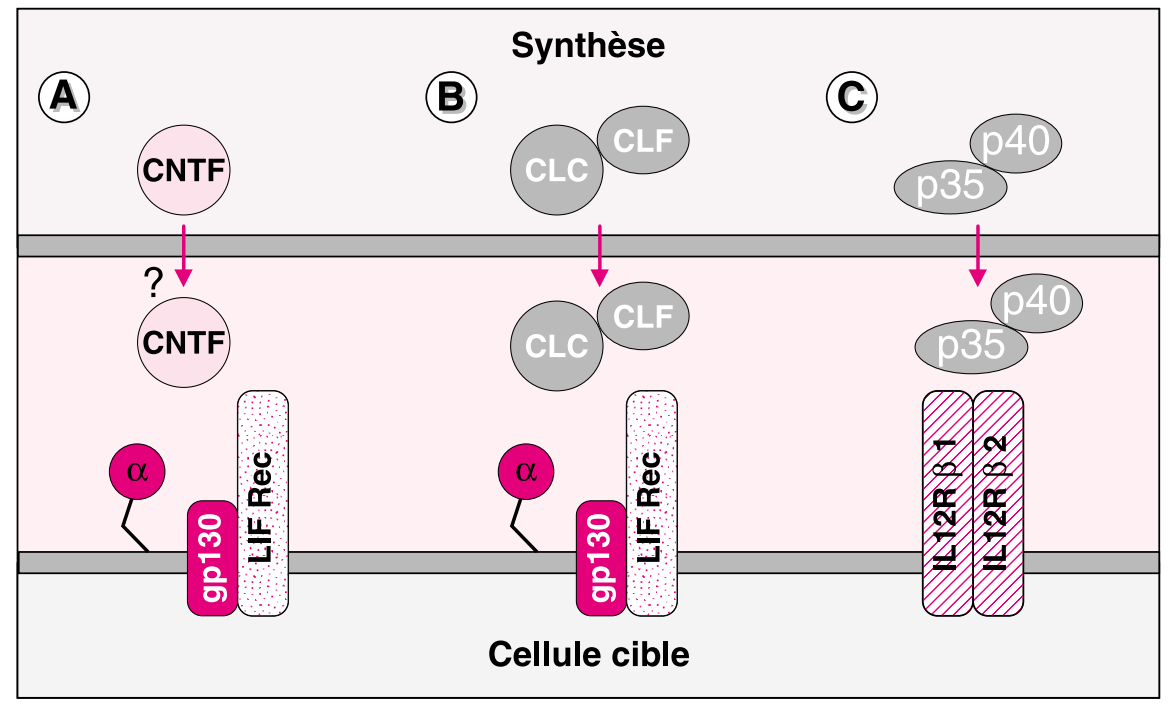

Figure 2. Mécanismes d'action du CNTF et de I'hétérodimère CLF/CLC. A. Le CNTF est libéré dans le milieu extracellulaire lors d'événements traumatiques. Le CNTF active ensuite son récepteur tripartite localisé sur le tissu cible. B. L'association de CLF avec CLC va permettre la libération de l'hétérocomplexe CLC/CLF qui va également agir sur le récepteur tripartite du CNTF. C. De façon voisine, I'IL-12 ne sera sécrétée que lors de l'association covalente de ses 2 sous-unités, p35 et p40. L'IL-12 agit ensuite sur son récepteur dimérique.

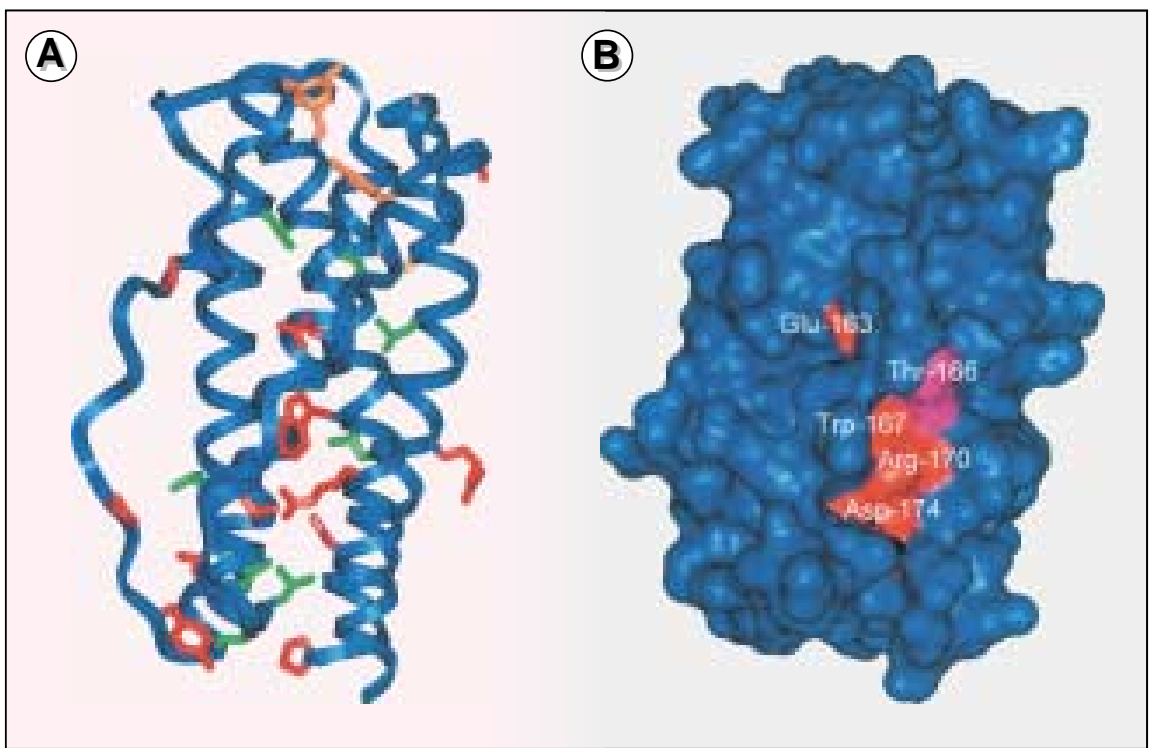

Figure 3. Structure de CLC et sa comparaison avec le CNTF. A. Représentation en ruban du modèle de CLC. Les chaînes latérales des résidus conservés dans CLC et CNTF sont représentés en couleur. Les leucines orientées vers l'intérieur de la protéine sont en vert. Les acides aminés impliqués dans le site "III" d'interaction avec LIFR, sont en orange. Les autres résidus conservés dans les 2 cytokines sont en rouge. B. Accessibilité aux solvants de CLC. Les résidus conservés dans l'hélice $D$ de CLC sont représentés en rouge et définissent le site "I" d'interaction avec le CNTFR.

$\mathrm{m} / \mathrm{s} \mathrm{n}^{\circ} 10$, vol. 16 , octobre 2000 ment ce complexe n'est pas doué d'activité biologique sur des cellules exprimant à leur surface uniquement gp130 et LIFR. Ce résultat indique que le complexe CLC/CLF n'est actif que sur des tissus exprimant à leur surface la forme membranaire du CNTFR. Le CNTFR soluble, présent dans le liquide céphalorachidien et le sérum, ne devrait donc pas pouvoir potentialiser la réponse CLC/CLF, contrairement à ce qui est observé pour le CNTF. Il n'est pas exclu que l'action de CLC/CLF fasse appel à une voie de transduction du signal activée spécifiquement par la forme membranaire de CNTFR.

D'une façon similaire à celle du CNTF, la fixation membranaire de CLC/CLF va induire la dimérisation et le recrutement des sous-unités de transduction gp130 et LIFR puis activer en aval les cascades de transduction du signal. L'analyse des niveaux de phosphorylation des résidus tyrosine des voies de signalisation indique une implication des kinases JAK1, JAK2 et Tyk2 et de la phosphatase SHP2. Le relais de l'information vers le noyau est notamment assuré par la molécule de transduction du signal STAT3, par la voie $\mathrm{Pi3}$ kinase/Akt ainsi que par les protéines Erk1, 2 appartenant à la voie des MAP kinases.

La cytokine purifiée a été testée in vitro dans différents systèmes biologiques. CLC/CLF active fortement les différentes voies de transduction du signal dans des lignées de neuroblastome qui expriment à leur surface le récepteur tripartite pour le CNTF. En revanche, elle est sans effet sur des lignées établies à partir de glioblastomes qui expriment gp130 et LIFR, mais pas CNTFR. La réintroduction dans la cellule de cette dernière sousunité permet alors de reconstituer une réponse fonctionnelle à CLC/CLF ainsi qu'au CNTF.

La mesure de la survie in vitro de motoneurones embryonnaires de rongeurs constitue un test biologique de référence pour déterminer l'activité des cytokines et facteurs de croissance susceptibles d'agir sur ce tissu. CLC/CLF permet effectivement d'obtenir un taux de survie des motoneurones isolés et purifiés à partir d'embryons de rat de 14,5 jours 
comparable à celui mesuré en présence de CNTF. Il est donc probable que CLC/CLF corresponde au second ligand du récepteur du CNTF, recherché par de nombreux groupes depuis plusieurs années. Il est important de mentionner l'identification de CLF de façon indépendante par un autre groupe [22]. L'inactivation du gène codant pour CLF conduit à un phénotype voisin de celui observé chez les animaux $C N T R^{/-}$avec mort périnatale des animaux, ce qui renforce encore l'analogie fonctionnelle entre CLC/CLF et le deuxième ligand pour le récepteur du CNTF. L'obtention de souris dont les deux gènes codant pour CNTF et CLC seraient invalidés devrait conduire à un phénotype identique à celui observé pour les souris dont le CNTFR a été invalidé. Il ne faut cependant pas exclure totalement la possibilité que d'autres ligands additionnels pour le CNTFR puissent exister.

Les activités neuroprotectrices in vitro et in vivo du CNTF ont conduit à des essais cliniques dans des pathologies neurodégénératives, comme la sclérose latérale amyotrophique ou la maladie de Huntington [23, 24] $(\mathrm{m} / \mathrm{s}$ 1997, $\left.n^{\circ} 6-7, p .901\right)$. Le mode d'administration de la cytokine est un point important pour éviter des effets secondaires. Sa diffusion lente utilisant des systèmes encapsulés et insérés au sein même des tissus cibles est une voie tout-à-fait prometteuse. CLC/CLF représente un deuxième ligand naturellement sécrété pour le CNTFR. La spécificité de son action est contrôlée à la fois au niveau du site de synthèse au travers de la fonction de transporteur membranaire de la partie CLF, mais également au niveau des tissus cibles puisque seule une forme membranaire de CNTFR autorise la réponse biologique. Son utilisation comme agent neuroprotecteur pourrait représenter un élément important dans le traitement des maladies neurodégénératives

\section{RÉFERENCES}

1. Lin LF, Mismer D, Lile JD, et al. Purification, cloning, and expression of ciliary neurotrophic factor (CNTF). Science 1989 ; 246: 1023-5.

2. Stöckli KA, Lottspeich F, Sendtner M, et nal distribution of rat ciliary neurotrophic factor. Nature 1989 ; 342 : 920-3.

3. Sendtner M, Gotz R, Holtmann B, Thoenen $H$. Endogenous ciliary neurotrophic factor is a lesion factor for axotomized motoneurons in adult mice. J Neurosci 1997 ; $17: 6999-7006$.

4. Sendtner M, Schmalbruch H, Stockli KA, Caroll P, Kreutzberg GW, Thoenen H Ciliary neurotrophic factor prevents degeneration of motor neurons in mouse mutant progressive motor neuronopathy. Nature 1992 ; 358 : 502-4.

5. Mitsumoto H, Ikeda K, Klinkosz B, Cedarbaum JM, Wong V, Lindsay RM. Arrest of motor neurone disease in wobbler mice cotreated with CNTF and BDNF. Science $1994 ; 265: 1107-10$.

6. Helgren M, Squinto SP, Davis HL et al. Trophic effect of ciliary neurotrophic factor on denervated skeletal muscle. Cell 1994 ; $76: 493-504$.

7. Guillet C, Auguste P, Mayo W, Kreher P, Gascan H. Ciliary neurotrophic factor is a regulator of muscular strength in aging. $J$ Neurosci 1999 ; 19 : 1257-62.

8. Takahashi R, Yokoii H, Misawa H, Hayashi M, Hu J, Deguchi T. A null mutation in the human CNTF gene is not causally related to neurological diseases. Nat Genet $1994 ; 7$ : 79-84.

9. DeChiara TM, Veisada R, Poueymirou WT, et al. Mice lacking the CNTF receptor, unlike mice lacking CNTF, exhibit profound motor neuron deficits at birth. Cell $1995 ; 83: 313-22$.

10. Taga T, KishimotoT. Gp130 and the interleukin-6 family of cytokines. Annu Rev Immunol $1997 ; 15$ : 797-819.

11. Shi Y, Wang W, Yourey PA, Gohari S, et al. Computational EST database analysis identifies a novel member of the neuropoietic cytokine family. Biochem Biophys Res Commun 1999 ; 262 : 132-8.

12. Senaldi G, Varnum BC, Sarmiento U, et al. Novel neurotrophin-1/B cell stimulating factor-3: a cytokine of the IL-6 family. Proc Natl Acad Sci USA 1999 ; 96 : 11458-63.

13. Bravo J, Heath JK. Receptor recognition by gp130 cytokines. EMBO J $2000 ; 19: 2399-$ 411.

14. Davis S, Aldrich TH, Valenzuela DM, et al. The receptor for ciliary neurotrophic factor. Science $1991 ; 253$ : 59-63.

15. Davis S, Aldrich TH, Stahl N, et al. LIFR beta and gp130 as heterodimerizing signal transducers of the tripartite CNTF receptor. Science 1993 ; 260 : 1805-8.

16. Stahl N, Boulton TG, Farrugella T, et al. Association and activation of Jak-Tyk kinases by CNTF-LIF-OSM-IL-6 beta receptor components. Science 1994 ; 263 : 92-5.

17. Stahl N, Farrugella TJ, Boulton TG, Zhong Z, Darnell JE, Yancopoulos GD. Choice of STATs and other substrates speci- fied by modular tyrosine-based motifs in cytokine receptors. Science 1995 ; 267 : 134953.

18. Elson GC, Graber P, Losberger C, et al. Cytokine-like factor-1, a novel soluble protein, shares homology with members of the cytokine type I receptor family. J Immunol $1998 ; 161$ : 1371-9.

19. Yoon C, Johnston SC, Tang J, Stahl M, Tobin JF, Somers WS. Charged residues dominate a unique interlocking topography in the heterodimeric cytokine interleukin12. EMBO J $2000 ; 19: 3530-41$.

20. Elson GC, Lelievre E, Guillet C, Chevalier S, Plun-Favreau H, Froger J, Suard I, BenoitdeCoignac A, Delneste Y, Bonnefoy JY, Gauchat JF, Gascan H. Cytokine-like factor-1 associates with cardiotrophin-1 like cytokine to form a functionnal heteromeric ligand for the CNTF receptor complex. Nat Neurosci $2000 ; 3$ : 867-72.

21. Davis S, Aldrich TH, Ip NY, et al. Released form of CNTF receptor $\alpha$ component as a soluble mediator of CNTF responses. Science 1993 ; 259 : 1736-9.

22. Alexander WS, Rakar S, Robb L, et al. Suckling defect in mice lacking the soluble haemopoietin receptor NR6. Curr Biol $1999 ; 9: 605-8$.

23. Aebischer P, Schluep M, Deglon N, et al. Intrathecal delivery of CNTF using encapsulated genetically modified xenogeneic cells in amyotrophic lateral sclerosis patients. Nat Med 1993 ; 2 : 696-9.

24. Bachoud-Levi A, Deglon N, Nguyen J, et al. Neuroprotective gene therapy for Huntington's disease using a polymer encapsulated BHK cell line engineered to secrete human CNTF. Hum Gene Ther 2000; 11 : 1723-9.

\section{Greg Elson \\ Jean-François Gauchat}

Centre d'immunologie Pierre Fabre, 5, avenue Napoléon-III, 74164 Saint Julienen-Genevois, France.

\section{Catherine Guillet \\ Éric Lelièvre Hugues Gascan}

Inserm EMI-U 9928, CHU d'Angers, 4, rue Larrey, 49033 Angers, France.

\section{TIRÉS À PART}

\section{H. Gascan.}

